\title{
L'angliciste de spécialité et son objet de recherche : contribution à la réflexion épistémologique
}

\section{Séverine Wozniak}

\section{(2) OpenEdition}

\section{Journals}

Édition électronique

URL : http://journals.openedition.org/asp/2721

DOI : 10.4000/asp.2721

ISSN : 2108-6354

Éditeur

Groupe d'étude et de recherche en anglais de spécialité

Édition imprimée

Date de publication : 15 mars 2012

Pagination : 25-37

ISSN : $1246-8185$

Référence électronique

Séverine Wozniak, «L'angliciste de spécialité et son objet de recherche : contribution à la réflexion épistémologique », ASp [En ligne], 61 | 2012, mis en ligne le 07 octobre 2014, consulté le 02 novembre 2020. URL : http://journals.openedition.org/asp/2721 ; DOI : https://doi.org/10.4000/asp.2721

Ce document a été généré automatiquement le 2 novembre 2020

Tous droits réservés 


\title{
L'angliciste de spécialité et son objet de recherche : contribution à la réflexion épistémologique
}

\author{
Séverine Wozniak
}

1 Un des postulats de la recherche en sciences sociales est que le chercheur exerce une influence sur son objet de recherche, directement ou indirectement, puisqu'il « importe dans ses observations les cadres théoriques qui le guident dans sa démarche » (Jodelet 2003 : 148). Pierre Bourdieu souligne la nécessaire dimension réflexive en sociologie, qu'il qualifie de « sociologie de la sociologie » :

Je crois que la sociologie de la sociologie est une dimension fondamentale de l'épistémologie de la sociologie. Loin d'être une spécialité parmi d'autres, elle est le préalable nécessaire de toute pratique sociologique rigoureuse. À mon avis, une des principales sources d'erreur dans les sciences sociales réside dans une relation incontrôlée à l'objet qui conduit à projeter cette relation non analysée dans l'objet de l'analyse. (Bourdieu \& Wacquant 1992 : 48, séminaire de Chicago)

2 Aussi il apparaît qu'il nous faut également mener ex ante cette réflexion centrale sur la position du chercheur dans notre discipline.

3 Afin de fonder notre réflexion sur la relation entre l'angliciste de spécialité et son objet de recherche, nous commençons par établir une synthèse de plusieurs études antérieures portant sur la question de la position du chercheur en sciences sociales. Nous prenons cette synthèse comme point de départ avant de la mettre en perspective avec le cas spécifique du chercheur en anglais de spécialité, dont nous définissons au préalable la position. Cette approche nous permet de confronter nos propres pratiques de recherche à la réflexion théorique dans le but de suggérer des hypothèses de travail. Nous précisons que la présente analyse a été menée dans le cadre d'une recherche doctorale qui vise à la caractérisation du domaine spécialisé des guides de haute montagne aux États-Unis et dans laquelle nous proposons un modèle de caractérisation des domaines spécialisés et de leurs discours (Wozniak 2011). 


\section{Posture du chercheur et réflexivité}

4 La question traitée porte essentiellement sur les liens entre le chercheur et les membres de la communauté spécialisée qu'il observe. Aujourd'hui, ce rapport est principalement fondé sur l'équivalence et la confiance; il s'est construit au fur et à mesure des avancées de la réflexion épistémologique dans le champ des sciences sociales. Harry Coenen (2001) a analysé la construction de ce rapport d'équivalence entre chercheurs et acteurs dans l'histoire de la recherche-action. Cette rechercheaction est un domaine approprié et facilement transposable à notre cas, puisque ce type de recherche (mêlant démarche ethnographique, recours à l'entretien, au questionnaire, etc.) induit une forte collaboration entre les chercheurs et la communauté observée. Dans les années 1970, certains chercheurs en sciences sociales (notamment P. Bourdieu et Anthony Giddens) ont proposé une lecture critique de l'approche traditionnelle de la position du chercheur. Cette approche traditionnelle, ou « consensus orthodoxe », se fonde sur trois principes selon A. Giddens :

- la société a ses besoins spécifiques propres, indépendamment des individus ;

- le modèle qui préside à l'exécution de la recherche scientifique sociale est le même que celui utilisé dans les sciences de la nature ;

- les chercheurs du domaine des sciences sociales sont mieux, et plus rapidement, en mesure de connaître les causes du comportement individuel que les personnes concernées. (Giddens $1996: 65$, cité dans Coenen $2001: 20$ )

5 D'une façon générale, l'évolution de la réflexion conduite dans les années 1970 s'est conclue par l'abandon de la perspective dualiste (chercheur/acteur, théorie/pratique...) au profit de schémas de lecture monistes et intégrateurs, telles les notions d'habitus et de champs, développées par P. Bourdieu. Il semble difficile aujourd'hui, dans le cadre d'une recherche en sciences sociales, de considérer le chercheur comme sujet distinct de l'objet de la recherche (l'acteur) ou en opposition à ce dernier. Le savoir du chercheur, comme celui de l'acteur, est fondamental, complémentaire dans le processus de recherche et déterminant pour la qualité des résultats dégagés. La perspective adoptée est beaucoup plus imbriquée : le chercheur est observateur mais aussi partie prenante, l'acteur n'est pas seulement «l'objet » observé mais il participe de la démarche de recherche.

6 Selon Harry Coenen, deux concepts centraux émergent dans la tentative de définition «normative » de la recherche : l'adéquation (aux attentes des acteurs et plus largement de la société) et l'explicitation (de la démarche de recherche). Le premier concept fait écho à la question polémique de l'adéquation à la demande sociale : il s'agit pour la connaissance d'émerger de la situation et d'y opérer un retour (cette adéquation est donc réciproque) : «Ce sont justement les connaissances scientifiques reposant sur ce savoir [celui fondé sur le bon sens] qui peuvent à leur tour servir à comprendre et à résoudre les problèmes de la pratique sociale » (2001: 23). L'explicitation est le second fondement de la démarche de recherche-action. En effet, le chercheur adopte une démarche scientifique critique par nature. Cependant, cette démarche ne peut avoir de sens que si ses objectifs et ses moyens sont explicités, donc rendus contrôlables (Coenen $2001: 24$ ).

7 Ainsi, la réflexivité apparaît comme un pivot central de la recherche en sciences sociales : le chercheur se doit sans cesse de soumettre ses hypothèses, sa démarche de recherche et ses conclusions à une lecture critique de sa propre position et de ses 
propres biais par rapport à son objet. Les sociologues éclairent la nature de cette réflexivité. Pour P. Bourdieu, cette réflexivité est nécessaire car, et c'est une particularité de la recherche en sciences sociales, le processus de recherche modifie l'objet de la recherche en ce que le chercheur exerce inévitablement une influence sur les acteurs et sur l'objet de sa recherche. Loïc Wacquant met en avant les trois types de biais « capables d'obscurcir le regard sociologique » identifiés par P. Bourdieu :

Le premier, qui a aussi été rappelé par d'autres, tient à l'origine et aux coordonnées personnelles (de classe, de sexe ou d'ethnie) du chercheur. C'est le biais le plus évident, et partant, le plus directement contrôlable au moyen de l'autocritique et de la critique mutuelle. Le second, bien moins souvent aperçu et discuté, est lié à la position que l'analyste occupe, non dans la structure sociale au sens large, mais dans le microcosme du champ académique, c'est-à-dire dans l'espace objectif des positions intellectuelles qui s'offrent à lui à un moment défini et, au-delà, dans le champ du pouvoir. Le biais intellectualiste, qui nous pousse à concevoir le monde comme un spectacle, comme un ensemble de significations demandant à être interprétées plutôt que comme des problèmes concrets appelant des solutions pratiques, est un biais beaucoup plus profond et beaucoup plus dangereux dans ses effets que ceux qui sont inscrits dans l'origine sociale et dans la position de l'analyste dans le champ universitaire : il conduit en effet à ignorer complètement ce qui fait la differencia specifica de la logique de la pratique. (Bourdieu \& Wacquant 1992:34-35)

8 Cette lecture appelle une explicitation de la position du chercheur indépendante de celle de l'acteur, instaurant en quelque sorte une rupture qui, selon H. Coenen, introduit une incompatibilité entre la position de P. Bourdieu et les postulats de la recherche-action (équivalence, réciprocité et interactivité). Selon P. Bourdieu, le chercheur est par nature au-dessus de l'objet, dans sa posture de savant, extérieur, voire au-dessus, du champ. Il nomme cet "ethnocentrisme de savant" " "biais" théoriciste ou intellectualiste» (Bourdieu \& Wacquant 1992 : 49-50). Le retour théorique qui s'opère à partir de la pratique provoque une rupture identifiée par Dick Pels dans son appropriation critique de la position de P. Bourdieu comme le «moment objectiviste » :

The complication which arises here is that the epistemological break or the 'objectivist moment', in which the sociologists [sic] constructs the (intellectual) field, is not itself reflexively recognized as a performative operation, as a definition of the situation that co-produces the situation that it describes, but still claims a privileged grounding in the objective reality of structured social relations. (2000:13)

Le chercheur doit alors délimiter le champ qu'il étudie et se positionner par rapport à ces limites ; il doit éviter de se considérer comme occupant une position privilégiée par rapport aux acteurs participant de la recherche. Au contraire, le chercheur doit privilégier un état de coopération maximal, afin de comprendre le groupe étudié au plus près, le processus de réflexion sur la posture ex ante et ex post permettant de neutraliser au maximum les biais.

10 À la lecture de ces conclusions théoriques, dans la perspective de notre travail de recherche, nous avons retenu qu'il est nécessaire de clarifier la position du chercheur « angliciste de spécialité » dans l'acception la plus classique du terme et de son statut. Cependant, il ne s'agit pas d'atteindre un point où la " méta-réflexion » sur la nature de la recherche et la position du chercheur serait contre-productive, mais de tenter d'établir, dans une certaine mesure, le point « d'où nous parlons » : 
Notwithstanding the risk of reflexive posturing, I still presume that it is both feasible and important to talk about something and simultaneously talk (at least a little) about the talking itself; and that it is better for your epistemological health to be reflexive rather than non-reflexive. (Pels $2000: 3$ )

11 L'objectif de cette démarche est de déduire les forces et les faiblesses de cette position et les principaux biais induits, afin d'en contrôler au maximum les externalités. P. Bourdieu note que le linguiste ne doit pas s'affranchir de la pensée réflexive :

En effet, aussi longtemps qu'ils ignorent la limite qui est constitutive de leur science, les linguistes n'ont d'autre choix que de chercher désespérément dans la langue ce qui est inscrit dans les relations sociales où elle fonctionne, ou de faire de la sociologie sans le savoir, c'est-à-dire avec le danger de découvrir dans la grammaire même ce que la sociologie spontanée du linguiste y a inconsciemment importé. (Bourdieu $2001: 60$ )

\section{L'angliciste de spécialité en contexte français}

Nous commençons par définir la position particulière du chercheur en anglais de spécialité, le travail de définition ex ante de la posture du chercheur étant, nous l'avons vu précédemment, une étape nécessaire à toute analyse en sciences sociales.Ainsi, nous tentons de caractériser l'angliciste de spécialité, ou plutôt, de définir ses caractéristiques « objectives ", son habitus, d'objectiver l'individu pour tenter d'établir un type général : «Parler d'habitus, c'est poser que l'individuel, et même le personnel, le subjectif, est social, collectif. L'habitus est une subjectivité socialisée » (Bourdieu \& Wacquant 1992 : 101). Nous voyons d'ailleurs en quoi cette démarche peut heurter les individus en tant que tels :

Ce qui exaspère, ou désespère, je crois, dans la notion d'habitus, c'est qu'elle incarne la mise en œuvre du mode de pensée génétique et générique qui menace l'idée même que les "créateurs" se font d'eux-mêmes, de leur identité, de leur « singularité ». (Bourdieu \& Wacquant 1992 : 108)

Cependant, puisque notre travail d'angliciste de spécialité vise à caractériser une communauté discursive professionnelle observée, il nous paraît important, en amont, de tenter de caractériser la communauté « observante ».

14 Tout d'abord, rappelons ce qui constitue l'objet de recherche des anglicistes de spécialité français : l'anglais de spécialité, « branche des études anglophones qui a pour objet l'étude des domaines spécialisés et du spécialisé en contexte anglophone » (Petit $2008: 23)^{1}$. Michel Petit place les analyses des discours et des styles spécialisés ${ }^{2}$ dans le cadre de la recherche en anglais de spécialité, distinguant deux volets constitutifs et indissociables. Le premier volet s'intéresse au processus de didactisation des connaissances sur les domaines spécialisés en contexte anglophone; le second porte sur l'élaboration de ces connaissances (Petit 2010:2). Les chercheurs français en anglais de spécialité s'accordent pratiquement tous aujourd'hui pour souligner que ce sont les domaines spécialisés (étudiés dans une perspective diachronique et/ou synchronique) et le spécialisé qui constituent désormais l'objet de recherche privilégié en anglais de spécialité :

[C]'est le spécialisé qui constitue l'objet spécifique de l'anglais de spécialité comme discipline et qui construit le principe unificateur de ses différents volets (étude de la langue et du discours spécialisés ; étude des domaines spécialisés) et différentes branches (« anglais» du droit, des mathématiques, du journalisme, de la politique, etc.). (Petit $2011: 184)$ 
15 Jacqueline Percebois avance également l'argument selon lequel les communautés discursives se trouvent à l'interface entre les domaines linguistique et disciplinaire ; ce dernier recoupant « toutes les connaissances impliquées par la communication et mises en œuvre dans les échanges discursifs» (2006: 67). Catherine Resche soumet une analyse du rapport entre langue et culture et rappelle leurs « rapports complexes d'interdépendance» (2009: 47). Cette perspective est également retenue par de nombreux chercheurs anglophones du champ de l'English for Specific Purposes: Ken Hyland, par exemple, remarque que le spécialisé est central et émerge des discours :

The principle of specificity receives strong theoretical endorsement from the philosophical perspective of social constructionism [...] and the critiques and extensions of it [...]. This stresses that disciplines and professions are largely created and maintained through the distinctive ways that members jointly construct a view of the world through their discourses. (2002:390)

Dans les paragraphes suivants, nous traitons de l'activité de recherche de l'angliciste de spécialité, et non de son activité d'enseignement, même si ces deux activités peuvent dans certains cas se recouper (cas d'un angliciste de spécialité travaillant sur la structure de l'article de recherche en anglais du génie mécanique enseignant à des doctorants en génie mécanique par exemple). Le rapport entre l'angliciste de spécialité, son objet d'enseignement et son objet de recherche n'a pas, à ce jour et à notre connaissance, fait l'objet d'une étude exhaustive, même si plusieurs chercheurs ont posé, dans une perspective d'enseignement des discours spécialisés, des jalons pour l'analyse de cette situation, comme le souligne Gail Taillefer, dans son étude sur l'évolution du métier d'enseignant d'anglais en secteur LANSAD (2008:59, voir aussi à ce sujet Mémet 2001, 2005 et 2008). Michel Van der Yeught remarque que, pour l'angliciste "généraliste" débutant dans un secteur spécialisé donné, l'objet de connaissance - le discours spécialisé - est souvent mal connu. Du moins, les éléments de connaissance de l'objet ne sont pas synthétisés :

[L]es anglicistes qui professent dans le secteur LANSAD se forment sur le tas et le plus souvent à l'aide de manuels produits à l'intention de leurs étudiants. Les savoirs dont ils ont un besoin urgent restent éparpillés dans des centaines d'articles de recherche encore trop déconnectés les uns des autres pour se combiner en savoirs homogènes. $(2010: 6)$

M. Van der Yeught met en avant le manque de formation initiale pour les anglicistes qui souhaitent se spécialiser en anglais de spécialité (de l'économie et de la gestion, du droit, de la médecine, etc.) ${ }^{3}$.

L'angliciste de spécialité est avant tout un angliciste, qui, grâce à sa formation initiale, est à même de mobiliser les outils de son champ disciplinaire: analyse "civilisationnelle ", littéraire et linguistique, de même que certaines compétences en traductologie. Il paraît important de relever ici que la démarche de recherche de l'angliciste de spécialité s'apparente à celle du chercheur en anglistique «conventionnelle»; elle s'inscrit bien dans le cadre paradigmatique des études du monde anglophone, le chercheur fait finalement appel à des concepts et des outils connus et établis de différentes disciplines et sous-disciplines connexes. Par définition, l'angliciste de spécialité, du fait de sa formation disciplinaire initiale, possède a priori une connaissance approfondie du contexte général dans lequel son terrain est inscrit : les États-Unis, le Royaume-Uni, etc. Il peut être un natif du pays ou bien y avoir séjourné pendant une longue période dans le cadre de ses études; il a de ce fait construit des connaissances synchroniques relevant de la vie politique, économique et 
sociale du pays, en plus des savoirs purement linguistiques. En outre, ayant suivi une formation d'angliciste conventionnel, il a souvent acquis des savoirs diachroniques se rapportant au cadre général de son terrain, incluant des éléments d'histoire, d'histoire des idées, de la société et de la langue, d'économie, de littérature...

Au-delà de cet acquis initial, l'angliciste de spécialité a souvent établi a minima des contacts préalables avec la communauté discursive professionnelle qu'il se propose de caractériser, c'est-à-dire avec les acteurs de son terrain de recherche, au moins en contexte français. Cette expérience constitue un avantage indéniable, là où de nombreux projets en sciences sociales portent sur des domaines orthogonaux aux intérêts et aux connaissances initiales du chercheur. Pour prendre pour exemples des travaux récents en anglais de spécialité, Anthony Saber a enseigné six ans à l'École navale de Lanvéoc avant de débuter son travail sur les milieux militaires états-uniens et leurs discours (Saber 2006); Hélène Laffont était en poste à l'Institut national des sciences appliquées de Toulouse lorsqu'elle travaillait sur l'anglais de l'ingénieur (Laffont 2005) tout comme Cédric Sarré, en poste à la Faculté des sciences de l'Université d'Orléans lorsqu'il a écrit sa thèse portant sur l'anglais de la biologie (Sarré 2010). Enfin, le travail de Marie-Agnès Détourbe porte sur le domaine spécialisé de l'enseignement supérieur britannique alors que cette chercheuse appartient à la communauté professionnelle de ce même domaine spécialisé en contexte français (Détourbe 2011). Ces contacts préalables avec le terrain permettent, d'emblée, de tester les critères de faisabilité et de pertinence de la recherche. C'est là, il nous semble, la valeur de recherche ajoutée de l'angliciste de spécialité : cette double compétence, garante d'une recherche de qualité, notamment lorsque le chercheur met en place une démarche ethnographique pour étudier le domaine spécialisé visé.

Les responsables d'une enquête par questionnaire doivent avoir acquis une compétence, un savoir-faire méthodologique, mais ils doivent aussi maittriser, en partie, la culture sociologique et la « culture » des acteurs sociaux. Seul ce mélange provoque une étincelle, l'intuition des idées. (de Singly 2005 : 30)

À notre avis cependant, cette position constitue un biais évident et ce biais nous conduit à nous interroger sur la façon d'aborder la grande majorité des domaines spécialisés professionnels, en particulier ceux pour lesquels il n'existe pas de formation universitaire. En d'autres termes, il convient de nous interroger sur la façon d'aborder le domaine lorsque le chercheur n'enseigne pas, en contexte français, aux professionnels en formation du domaine spécialisé visé.

\section{L'angliciste de spécialité et son objet de recherche}

Comme énoncé plus haut, la présente analyse a été menée dans le cadre d'une recherche doctorale pour laquelle nous avions justement choisi de nous intéresser à un domaine spécialisé entrant dans ce cadre: celui des guides de haute montagne aux États-Unis. Nous avions une connaissance préalable du domaine spécialisé de l'alpinisme en tant que pratiquante amatrice et cet intérêt nous a amenée à penser qu'il paraissait, a priori, correspondre à un domaine pertinent pour pouvoir mettre en œuvre notre protocole méthodologique. Aussi, au commencement de notre recherche doctorale, nous disposions de connaissances préalables et d'un réseau familial et amical très intégré, en contexte français, dans le domaine spécialisé que nous nous proposions d'étudier ${ }^{4}$. 
22 Afin de pénétrer le domaine spécialisé ciblé, d'abord en contexte francophone puis en contexte anglophone, dans un premier temps, nous avons décidé de nous rapprocher des guides de haute montagne français, et plus particulièrement des institutions formant l'exercice professionnel des guides et l'encadrant. Nous avons fait l'hypothèse que cette démarche nous permettrait de nous familiariser avec le domaine spécialisé et de développer notre connaissance de la profession en général, de façon à être à même, dans un second temps, d'établir des contacts avec les institutions équivalentes aux États-Unis et d'identifier les caractéristiques propres à la pratique du métier en contexte états-unien. Pour atteindre notre objectif, nous nous sommes appuyée sur notre expérience d'enseignante d'anglais de spécialité pour proposer une étude des besoins langagiers des guides français, par le biais d'une analyse de besoins ${ }^{5}$. L'analyse des besoins langagiers est un outil d'évaluation permettant la prise de décision en matière d'enseignement /apprentissage des langues et de politique de développement des compétences langagières, car elle permet de définir des objectifs d'apprentissage appropriés, le contenu des programmes, les méthodes d'enseignement et d'évaluation ainsi que les ressources et le matériel pédagogique à utiliser (Brindley $2004: 438$ ). M. Van der Yeught souligne les limites de l'utilisation de cet outil :

Le recours à l'analyse des besoins langagiers (needs analysis) et aux spécialistes du domaine semble devoir s'imposer partout comme une solution indiscutable sans qu'aucune règle de décision issue du «spécialisé » propre aux langues elles-mêmes n'ait été envisagée. [... Ce sont des positions] pleinement justifiées lorsque la langue est destinée à une application immédiate, dans ce qui pourrait être appelée une "synchronie contemporaine » des besoins, mais elles trouvent rapidement leurs limites dès qu'il s'agit de transmettre la langue-culture spécialisée de façon approfondie. (2009: 44-45)

Cependant, cet outil nous a paru adapté à notre objectif, car notre hypothèse de recherche était que, grâce à ce projet, nous réussirions à pénétrer ce domaine spécialisé relativement fermé, afin de comprendre les enjeux de la formation et de la pratique du métier dans un contexte français dans un premier temps et, dans un second temps, aux États-Unis. Le recours à l'analyse des besoins langagiers a été finalement retenu, mais d'autres possibilités sont offertes à l'angliciste de spécialité qui souhaite aborder un domaine spécialisé difficile d'accès. Ainsi, si la suggestion de cette analyse des besoins a été une première étape, au fil du temps de la recherche, il a été question de nous associer à un travail de recension des termes de l'alpinisme, dans le but de renouveler un glossaire multilingue utilisable par les professionnels français. Ainsi, l'angliciste de spécialité peut aborder son terrain en mettant en avant, le cas échéant, son expertise de terminologue. Cette expertise lui permet d'ailleurs d'aller bien au-delà de la simple dénomination des concepts et de leurs liens au sein d'un sous-domaine pour montrer aux spécialistes eux-mêmes les évolutions de certains concepts, de leur signaler comment la langue peut aider à débusquer des changements de paradigmes au sein de leur domaine, changements qu'eux-mêmes ont parfois du mal à percevoir. L'angliciste de spécialité peut également proposer un retour ex post sur sa recherche concernant un domaine spécialisé anglophone auprès des professionnels français, généralement intéressés par les détails de la formation ou de l'organisation du domaine, ou bien encore par les conditions de pratique des professionnels dans un contexte étranger.

L'un des postulats de cette hypothèse est que les compétences professionnelles de l'enseignant ouvrent au chercheur des connaissances sur son objet de recherche, ce qui apparaît comme un renversement par rapport au postulat traditionnel selon lequel 
l'activité de veille scientifique et de construction de la connaissance par le chercheur nourrit la pratique de l'enseignant. Dans notre cas précis, nous mobilisons les compétences développées en tant qu'enseignante d'anglais de spécialité dans le domaine spécialisé de l'économie et de la gestion - compétences transférables à d'autres domaines - pour contribuer à l'avancée de notre projet de recherche sur un autre domaine spécialisé (l'anglais de l'alpinisme) sans perspective pédagogique ou didactique, puisque notre objectif n'est pas d'enseigner le discours spécialisé de notre domaine de recherche, mais de contribuer, plus largement, à la construction des dispositifs et des méthodologies de connaissance des domaines spécialisés. Une fois que nous avons réussi à établir ces premiers contacts avec les spécialistes du domaine, a émergé la question du lien entre le chercheur et les spécialistes.

Plusieurs auteurs ont abordé cette question de l'intensité du rapport et du degré d'intégration souhaitable entre le linguiste de spécialité et son domaine de recherche. En effet, le chercheur se doit de maintenir une certaine distance avec le domaine étudié, de considérer les pratiques et les faits sociaux comme des choses, de ne pas y être trop impliqué afin d'obtenir des résultats valides, comme l'énonçait dès l'origine Émile Durkheim, dans Les règles de la méthode sociologique (préface de la seconde édition, 1937 : xiii). Il s'agit là d'un prérequis complexe pour le chercheur : connaître a priori le domaine implique parfois également d'avoir des connaissances stéréotypées, ou même erronées, et être engagé «affectivement ». Ce prérequis est un atout évident, mais il peut également constituer un biais, non moins évident, que le chercheur doit garder à l'esprit pendant toutes les étapes de son travail. Pour revenir à la question du lien entre l'angliciste de spécialité et son domaine de recherche, nous remarquons que certains chercheurs ont abordé la question mais en semblant privilégier l'angle didactique, s'attachant à trouver comment l'enseignant d'anglais de spécialité pouvait acquérir le niveau de connaissance du domaine lui permettant de répondre aux besoins de ses apprenants.

Shaeda Isani s'interroge sur le mode approprié d'«acquisition par l'enseignant de langue d'une compétence de culture professionnelle qui n'est pas la sienne » (2004: $15)$; elle souligne les problèmes d'accès au savoir, et rappelle la "problématique récurrente du degré d'investissement de l'enseignant de langues de spécialité dans la constitution de son capital de savoir disciplinaire» (2004: 15). Dans son ouvrage portant sur le français sur objectifs spécifiques, Denis Lehmann prend comme point de départ le fait que le linguiste de spécialité, grâce à sa formation et sa propre culture professionnelle, a souvent eu des contacts limités avec d'autres milieux professionnels, en particulier l'entreprise privée :

Toute information concernant les comportements professionnels ayant cours dans tel pays, telle profession, tel groupe social, telle entreprise, revêt un intérêt d'autant plus grand que le « monde du travail », comme on dit parfois en pensant à celui de l'usine, du commerce, de l'entreprise en général, est le plus souvent parfaitement étranger à l'enseignant, y compris dans son propre pays, surtout l'enseignant littéraire. Il en ignore les règles et les codes, ce monde est pour lui un mystère quasiment indéchiffrable. On voit d'ailleurs bien là la dimension du problème: l'enseignant de français spécialisé devra d'abord mener à bien une acculturation personnelle (intra-culturelle si l'on veut) avant d'être en mesure de favoriser chez les apprenants avec qui il travaillera une autre acculturation, interculturelle celle-là. (1993: 13)

La relation entre l'enseignant d'anglais de spécialité et son domaine d'enseignement a donc fait l'objet d'une réflexion approfondie, mais qu'en est-il de la relation entre le 
chercheur en anglais de spécialité et le domaine spécialisé qu'il souhaite caractériser? John Humbley a considéré cette situation par le biais de la relation du terminologue et du spécialiste du domaine considéré, en soulignant le rôle d'interface du terminologue, au-delà des activités terminographiques $(1998: 148)$ et en s'interrogeant sur le degré d'expertise nécessaire pour le terminologue dans le domaine de spécialisation qu'il étudie :

Chacun conserve sa sphère de compétence, des sphères qui se recoupent largement tout en restant spécifiques. On peut citer le cas de la terminologue Zélie Guével, aujourd'hui professeur à l'Université Laval à Québec, mais pendant de longues années terminologue à l'ordre des comptables du Québec. Interrogée sur ses compétences en comptabilité, elle insiste toujours sur le fait qu'elle n'est pas devenue comptable, mais qu'elle est capable de dialoguer avec les comptables, de leur poser des questions et de comprendre les réponses, de traduire n'importe quel texte de comptabilité. Ceci me semble être ce qu'on peut exiger du terminologue surtout dans le contexte de la formation. (1998:140)

Nous exposons, dans cet article, les hypothèses de travail préalables que nous avons retenues pour mener notre recherche sur le domaine spécialisé des guides de haute montagne aux États-Unis. Nous avons commencé par présenter l'état de la réflexion sur la position du chercheur en sciences sociales et l'importance des pratiques réflexives avant de nous attacher plus particulièrement à définir le cas spécifique du chercheur en anglais de spécialité. Pour finir, nous proposons d'élargir la conclusion de J. Humbley aux anglicistes de spécialité et de rappeler que le chercheur en anglais de spécialité se doit également de développer une connaissance au second degré du domaine spécialisé qu'il se propose de caractériser. L'élaboration de cette connaissance peut être favorisée, entre autres, par la construction d'un rapport d'équivalence et de confiance avec les acteurs du domaine spécialisé, en contexte français et en contexte anglophone. Ce rapport d'équivalence (à ne pas confondre avec l'égalité, car la relation est très généralement asymétrique à certains moments de la recherche) entre le chercheur et les acteurs du domaine spécialisé est en partie fondé sur une relation de confiance. C'est la construction commune de la symétrie dans le rapport d'équivalence qui permet la mise en place de cette relation de confiance, essentielle à la réussite du projet de recherche, notamment dans la perspective de la mise en œuvre d'une démarche ethnographique.

Il nous a semblé, effectivement, que l'angliciste de spécialité, en tant que spécialiste d'un domaine, peut inscrire sa recherche dans une relation d'équivalence: ses compétences variées correspondant bien souvent à une demande sociale dans un monde où l'anglais est la lingua franca professionnelle. L'établissement de ce rapport de confiance et de symétrie participe de la création de valeur ajoutée de l'angliciste de spécialité et lui permet d'éviter le biais intellectualiste, pour comprendre les attentes de la communauté étudiée et faciliter ainsi sa bonne intégration au domaine qu'il se propose de caractériser, en contexte francophone puis en contexte anglophone.

L'auteure, qui reste cependant seule responsable des éventuelles erreurs et imprécisions, remercie Mme le Professeur Catherine Resche pour sa relecture attentive et ses commentaires avisés sur ce travail. 


\section{BIBLIOGRAPHIE}

Bourdieu, Pierre. 2001 «L'économie des échanges linguistiques ». In Bourdieu, Pierre, Langage et pouvoir symbolique. Paris : Le Seuil, 57-151.

Bourdieu, Pierre \& Loïc Wacquant. 1992. Réponses : Pour une anthropologie réflexive. Paris : Le Seuil. Brindley, Geoff. 2004. « Needs analysis ». In Byram, Michael (dir.), Routledge Encyclopedia of Language Teaching and Learning. Londres \& New York, NY : Routledge, 438-441.

Coenen, Harry. 2001. « Recherche-action : rapports entre chercheurs et acteurs ». Revue internationale de psychosociologie 1-2, $\mathrm{n}^{\circ} 16-17,19-32$.

Détourbe, Marie-Agnès. 2011. «Contribution à la caractérisation des pratiques d'évaluation dans le monde de l'enseignement supérieur britannique envisagé comme domaine spécialisé ». Thèse de doctorat, Université Bordeaux Segalen.

Durkheim, Émile. 1937. Les règles de la méthode sociologique. Paris : Presses Universitaires de France.

Giddens, Anthony. 1996. In Defense of Sociology. Cambridge : Polity Press.

Humbley, John. 1998. « Le terminologue et le spécialiste de domaine ». ASp 19-22, 137-149.

Hyland, Ken. 2002. « Specificity revisited: How far should we go now? ». English for Specific Purposes 21, 385-395.

Isani, Shaeda. 2004. «Compétence de culture professionnelle : définition, degrés et didactisation ». ASp 43-44, 6-21.

Jodelet, Denise. 2003. « Aperçus sur les méthodologies qualitatives ». In Moscovici, Serge \& Fabrice Buschini (dir.), Les méthodes des sciences humaines. Paris : Presses Universitaires de France, 139-162.

Laffont, Hélène 2005. «Contribution à la caractérisation de l'anglais pour l'ingénieur ». Thèse de doctorat, Université Victor Segalen Bordeaux 2.

Lehmann, Denis. 1993. Objectifs spécifiques en langue étrangère. Les programmes en question. Paris : Hachette.

Mémet, Monique. 2001. «Bref historique de l'enseignement et de la recherche en anglais de spécialité en France : de l'anglais pour non-spécialistes à l'anglistique du secteur LANSAD ». In Mémet, Monique \& Michel Petit (dir.), L'anglais de spécialité en France, Mélanges en l'honneur de Michel Perrin. Bordeaux : GERAS Éditeur, 309-319.

Mémet, Monique. 2005. « Développement de l'enseignement et de la recherche en anglais de spécialité : avancées théoriques et applications pédagogiques ». Les Langues modernes 1, 31-38.

Mémet, Monique. 2008. « Aspects de la recherche en anglais de spécialité en France ».Cahier du CIEL 2007-2008, 13-36. <http://www.eila.univ-paris-diderot.fr/recherche/clillac/ciel/cahiers/ 2007-2008>.

Pels, Dick. 2000. « Reflexivity: One step up ». Theory, Culture and Society 17/3, 1-25.

Percebois, Jacqueline. 2006. « Les communautés discursives à l'interface du linguistique et du disciplinaire ».In Greenstein, Rosalind (dir.), Langues et cultures : une histoire d'interface ? Paris : Publications de la Sorbonne, 67-89. 
Petit, Michel. 2005. «La notion de style spécialisé et les divers types de "spécialisé” ». In Sborník Príspevku Z Konference Profilingua 2005 (Actes de la Conférence Profilingua 2005). Pilsen :

Zapadoceska univerzita v Plzni, 140-144.

Petit, Michel. 2008. « Once more unto the breach, dear friends ». ASp, numéro spécial « Les trente ans du GERAS », 21-24.

Petit, Michel. 2010. «Le discours spécialisé et le spécialisé du discours : repères pour l'analyse du discours en anglais de spécialité ». E-rea [En ligne], 8/1 été 2010, mis en ligne le 21 septembre 2010, consulté le $1^{\text {er }}$ février 2011. <http://erea.revues.org/1400>.

Petit, Michel. 2011. «En guise de postface : le style spécialisé comme objet d'étude et domaine de recherche ». Bulletin de la Société de Stylistique Anglaise 35, 179-185.

Resche, Catherine. 2009. « Le discours du président de la Réserve fédérale américaine : langue de culture, culture de la langue ? ». In Greenstein, Rosalind (dir.), Langue et culture : mariage de raison? Paris : Publications de la Sorbonne, 45-93.

Saber, Anthony. 2006. « Les milieux militaires américains et leurs discours ». Thèse de doctorat, Université Victor Segalen Bordeaux 2.

Sarré, Cédric. 2010. « Approche collaborative de l'apprentissage de l'anglais de spécialité à distance dans un environnement intégrant les TIC : cas de l'anglais de la biologie ». Thèse de doctorat, Université du Havre.

Singly (De), François. 2005. L'enquête et ses méthodes, le questionnaire. Paris : Armand Colin, coll. «128».

Taillefer, Gail. 2008. « Transformations, évolution : un regard sur la dynamique de notre métier ». Les Cahiers de l'APLIUT 27/2, 49-65.

Van der Yeught, Michel. 2009. «Structurer l'anglais de la bourse : une approche des langues de spécialité par la spécialité ». Synthèse présentée pour l'obtention de l'habilitation à diriger des recherches, Université Victor Segalen Bordeaux 2.

Van der Yeught, Michel. 2010. « Éditorial ». ASp 57, 1-10.

Wozniak, Séverine. 2006. «To boldly go where no man has gone before: Mon premier poste LANSAD à l'université ». Les Langues modernes 3, 28-33.

Wozniak, Séverine. 2010. «Language needs analysis from a perspective of international professional mobility: The case of French mountain guides ». English for Specific Purposes 29/4 (ESP Research in Europe), 243-252.

Wozniak, Séverine. 2011. « Contribution à la caractérisation de l'anglais de l'alpinisme par l'étude du domaine spécialisé des guides de haute montagne états-uniens ». Thèse de doctorat, Université Bordeaux Segalen.

\section{NOTES}

1. Pour une étude de l'évolution de la définition de la notion d'anglais de spécialité selon Michel Petit, voir Wozniak $2011: 6-7$.

2. «faisceau des traits d'expression dont la mise en œuvre contribue à constituer en ensemble discursif ou discours spécialisé une série de productions langagières réunies par leur rapport constitutif avec un même milieu spécialisé » (Petit 2005 : 141).

3. Sur ce point, voir également Wozniak 2006. 
4. Je souhaite mentionner ici la participation à notre recherche de Thomas Emonet, aspirant guide de haute montagne, qui a pré-testé et commenté le questionnaire d'analyse des besoins langagiers des guides juniors et a été emporté par une avalanche dans le massif du mont Blanc en février 2010.

5. Une partie des résultats de cette recherche a fait l'objet d'une publication (Wozniak 2010).

\section{RÉSUMÉS}

Cet article propose une contribution à la réflexion épistémologique sur la relation entre l'angliciste de spécialité et son objet de recherche, l'anglais de spécialité. Prenant pour point de départ une analyse de la contribution des sciences sociales à cette question, en particulier la portée de la dimension réflexive en sociologie telle qu'énoncée par Pierre Bourdieu, l'auteure commence par définir la position du chercheur en anglais de spécialité par rapport à son objet de recherche. Cette question est d'autant plus pertinente que sont prises en compte les recherches portant sur des domaines spécialisés qui ne font pas l'objet d'un enseignement universitaire en France et qui sont, de ce fait, plus difficiles à appréhender pour l'angliciste de spécialité de l'enseignement supérieur. Le dernier temps de cet article s'attache à aborder cette question, en montrant notamment comment le chercheur en anglais de spécialité peut approcher le domaine spécialisé visé, même lorsque ce dernier ne relève pas de la sphère universitaire.

This paper aims at contributing to the epistemological reflection on the relationship between French ESP researchers and their research object: English for Specific Purposes. The author first defines the position of French ESP researchers in relation to their research object, using the insights afforded by social science, and more particularly reflexive practice as highlighted by Pierre Bourdieu. Such an approach is particularly adapted to the analysis of specialized domains that do not correspond to any academic discipline and are thus more difficult to comprehend for French ESP researchers. The paper examines this question at greater length by showing how researchers can rely on their experience as language specialists to compensate for the unavailability of any disciplinary framework in the targeted specialized domain to guide their steps.

\section{INDEX}

Mots-clés : anglais de spécialité, domaine spécialisé, épistémologie, objet de recherche, réflexivité, sciences sociales

Keywords : English for Specific Purposes, epistemology, reflexivity, research object, social science, specialized domain

\section{AUTEUR}

\section{SÉVERINE WOZNIAK}

Séverine Wozniak, docteure en études anglaises, est professeure agrégée d'anglais à la Faculté d'économie de Grenoble (Université Pierre-Mendès-France). Elle mène ses recherches, qui 
portent sur l'analyse des domaines spécialisés et de leurs discours en contexte états-unien, au sein de l'ILCEA/GREMUTS (Université Stendhal Grenoble III). severine.wozniak@upmf-

grenoble.fr 